BULLETIN (New Series) OF THE

AMERICAN MATHEMATICAL SOCIETY

Volume 37, Number 2, Pages 141-154

S 0273-0979(00)00857-0

Article electronically published on January 21, 2000

\title{
VARIATIONS ON CONSERVATION LAWS FOR THE WAVE EQUATION
}

\author{
CATHLEEN SYNGE MORAWETZ
}

\begin{abstract}
The first part of this paper, presented as an Emmy Noether lecture in connection with the ICM in Berlin in August 1998, gives some examples of using Noether's theorem for conservation laws for Tricomi-like equations and for the wave equation. It is also shown that equations which are semilinear variations of the wave equation can very often be handled similarly. The type of estimate obtained can even be used to get otherwise unobtainable local estimates for regularity.

The fourth part is an introduction to the relation of black holes to the wave equation mainly showing the results of D. Christodoulou. His results use much more difficult estimates not corresponding at all to those in the first part of the paper.
\end{abstract}

\section{INTRODUCTION}

It is a very, very great honor to give the Emmy Noether lecture here in Berlin at the time of the International Congress of 1998, the last of this century. I know that this honor to women would have been impossible forty years ago. To the best of my knowledge sometimes the ICM gave special attention to small countries but not to women or certain minorities. Also my great hope is that say thirty years from now the need for such a lecture will have evaporated. But now I wish to thank my sponsors, the Association for Women in Mathematics and the European Women in Mathematics, as well as the organizers of the Congress.

Mathematics is the queen of the sciences, and Emmy Noether is the queen of mathematics. I never met her, but she was a good friend of my mentors, Kurt Friedrichs and Richard Courant, who spoke to me about her many times. A nonreligious Jewish refugee from Nazi Germany, she died at the age of fifty-four in the USA. She did not live to receive either the job or the accolades that she deserved for her profound work in developing algebra. On the other hand, she did not live to know of the horrors that awaited the Jews of Europe, including many of her old friends; or the terrors of Stalinism, into which her brother disappeared; or the appalling destruction of the cities of Europe and of many of the friends of her youth.

Here I would like to make use of another of her contributions to mathematics, this one not to algebra but to partial differential equations. According to what I have heard, it was not one that she particularly liked. It followed on some work on invariants that had involved some exceedingly tedious calculations. But it has generated a number of important physical applications.

Received by the editors July 1, 1999, and in revised form October 6, 1999.

2000 Mathematics Subject Classification. Primary 35Lxx, 35Mxx, 35Qxx, 83Cxx.

Key words and phrases. Nonlinear, semilinear, p.d.e., Tricomi equation, black hole. 


\section{Noether's TheOREM AND The TRICOMi EQUATION}

Suppose we have a system of equations derivable from a Lagrangian. Noether's theorem [16] for conservation laws states that:

To every change of variables (independent or dependent) that leaves the action of a system - that is, the integral of the Lagrangian - invariant there corresponds a conservation law for the system.

In other words, to the group of invariant transformations there corresponds a group of conservation laws.

As a first example we will derive Noether's theorem for Tricomi-like equations, that is, for

$$
K(y) u_{x x}+u_{y y}=0 \quad \text { where } \quad y K(y) \geq 0,
$$

in a domain $\Omega$ that is for the time being arbitrary. This equation behaves like the wave equation (hyperbolic) for $y<0$ and like Laplace's equation (elliptic) for $y>0$. The action of the Lagrangian is, not surprisingly,

$$
A=\int_{\Omega}\left(K u_{x}^{2}+u_{y}^{2}\right) d x d y .
$$

It is invariant under a shift in $x$.

Let us compute the first variation, which must vanish,

$$
\begin{gathered}
0=\delta A=\int_{\Omega}\left(2 K u_{x} \delta u_{x}+2 u_{y} \delta u_{y}\right) d x d y+ \\
\int_{\partial \Omega}\left(K u_{x}^{2}+u_{y}^{2}\right) \delta n d s
\end{gathered}
$$

where $\delta n$ is the variation in the normal, $\delta u$ in $u$. Integrating the first term by parts, we find:

$$
\begin{aligned}
0=- & \int_{\Omega} 2\left(K u_{x x}+u_{y y}\right) \delta u d x d y \\
& +\int_{\partial \Omega}\left\{2\left(K u_{x} d y-u_{y} d x\right) \delta u+\left(K u_{x}^{2}+u_{y}^{2}\right) \delta n\right\} d s .
\end{aligned}
$$

The first term must vanish since $\delta u$ is arbitrary. This gives us as expected the differential equation (2.1). We use next the invariance with respect to $x$, on the boundary term,

$$
x \rightarrow x+\epsilon, \quad y \rightarrow y, \quad u(x, y) \rightarrow u(x-\epsilon, y),
$$

or $\delta u=-u_{x} \epsilon$, and $\delta n d s=\epsilon d y$. The vanishing of the variation of the action reduces to

$$
0=\int_{\partial \Omega}-2\left(K u_{x} d y-u_{y} d x\right) u_{x}+\left(K u_{x}^{2}+u_{y}^{2}\right) d y
$$

for arbitrary $\partial \Omega$. Or,

$$
0=\int_{\partial \Omega}-2 u_{x} u_{y} d x+\left(K u_{x}^{2}-u_{y}^{2}\right) d y .
$$

Since $\partial \Omega$ is an arbitrary curve, this is the conservation law. Note that the Noether conservation law "comes out of the boundary" as it does generally. Like every $2 \mathrm{D}$ conservation law it implies the existence of a function $U$ defined by

$$
U_{x}=-2 u_{x} u_{y}, \quad U_{y}=K(y) u_{x}^{2}-u_{y}^{2} .
$$


The function $U$ turns out to be very useful for uniqueness theorems for a wide range of domains that straddle the line $y=0$, where the equation changes type.

$U$ has three important properties:

(A) If $u=0$ on a curve $\Gamma$, then

(B)

$d y d U \geq 0$ on $\Gamma$ where $y \geq 0$,

and $\quad d y d U \geq 0$ on $\Gamma$ where $y<0$ and $|d x / d y|>\sqrt{-K}$.

$d y d U \leq 0$ for $y \leq 0$ on

characteristics, $d x / d y= \pm \sqrt{-K}$.

(C) $U$ satisfies a strong maximum principle in $y>0$.

The first two properties, (A) and (B), easily fall out of the definition of $U$.

Proof of $(C)$. In $y>0$, using (2.1), we find

$$
\begin{aligned}
K(y) U_{x x}+U_{y y} & =\frac{1}{2} \frac{K^{\prime}(y)}{K(y)}\left(\alpha U_{x}+\beta U_{y}\right) \\
\text { where } \quad \alpha & =1+U_{y}\left(K U_{x}^{2}+U_{y}^{2}\right)^{-1 / 2} \text { and } \\
\beta & =K U_{x}\left(K U_{x}^{2}+U_{y}^{2}\right)^{-1 / 2}
\end{aligned}
$$

are both bounded and measurable. Since $K>0$ we can use standard elliptic theory to complete the proof. We are assuming here that $K, u$ and any relevant boundary have sufficient smoothness.

Uniqueness theorems can be derived from (A), (B) and (C). To prove uniqueness we need just to prove that the corresponding homogeneous problem has only the zero solution $u \equiv 0$.

We consider the so-called Frankl problem:

The solution $u$ satisfies (2.1) in $\Omega$ where $\Omega$ is a domain (see Figure 1 ) bounded by $\Gamma_{0}+\Gamma_{1}+\Gamma_{-}$. Here $\Gamma_{0}$ lies in $y \geq 0$ and is convex. $\Gamma_{1}$ is a curve satisfying the Frankl condition $\left|\frac{d x}{d y}\right|>\sqrt{-K}, \frac{d x}{d y} \leq 0$, and $\Gamma_{-}$is a characteristic $\frac{d x}{d y}=-\sqrt{-K}$. The boundary condition is:

$$
u=0 \quad \text { on } \quad \Gamma_{0}+\Gamma_{1} .
$$

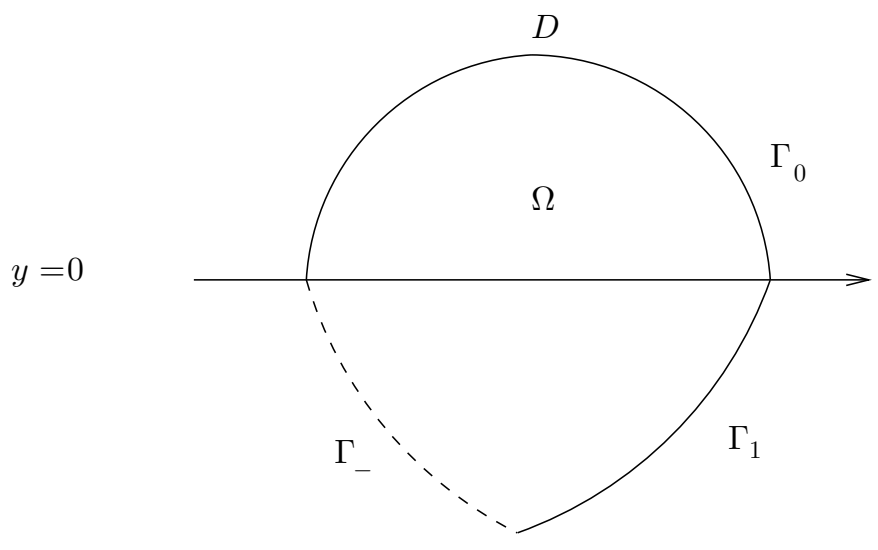

FiguRE 1 


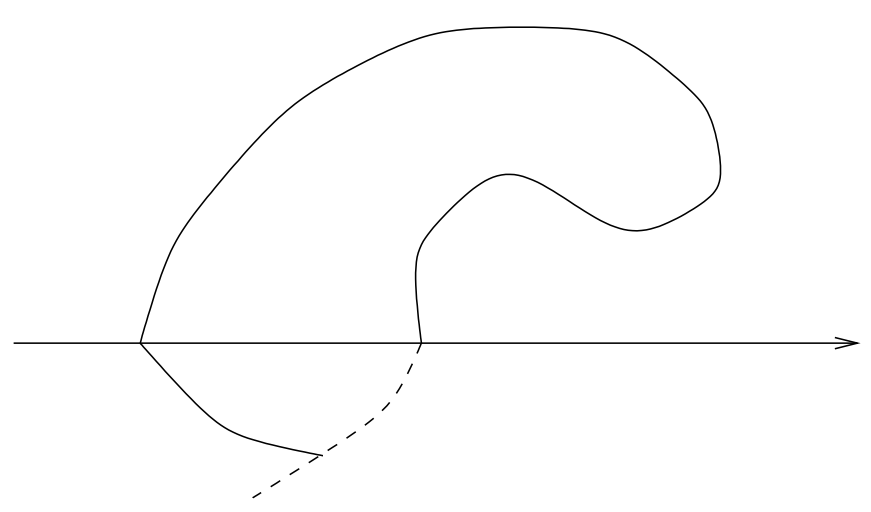

FiguRE 2

We assume $u, K$ and $\partial \Omega$ are smooth.

Theorem 1. [12]

$$
u \equiv 0 \quad \text { in } \partial \Omega .
$$

Proof. At $D$, the unique point on $\partial \Omega$ where $y$ is maximum, we have $U_{y}=-u_{y}^{2} \leq 0$. Hence $U$ does not have its maximum in $\bar{\Omega} \cap\{y>0\}$ at $D$ by the strong maximum principle (C). By (A) and (C), $U$ does not have its maximum in $\bar{\Omega} \cap\{y>0\}$. Thus $U$ has its maximum on $y \leq 0$. By applying (A) for $y \leq 0$ and (B) it is easily seen that $U$ must be identically constant, and thus by the definition of $U$ along with the boundary condition (2) we have $u \equiv 0$.

Of course much more general domains $\Omega$ can be constructed, but they do not include the very simple domain of Figure 2. One can, however, assert that for an arbitrary piecewise smooth elliptic boundary the solution space of the homogeneous problem is finite dimensional.

\section{THE WAVE EQUATION}

The next application of Noether's theorem is to the wave operator:

$$
\square=\frac{\partial^{2}}{\partial t^{2}}-\Delta \text {. }
$$

This operator comes from the action of a Lagrangian that is invariant under translation, stretching, rotation on a sphere and, most useful of all as it turns out, under the so-called conformal transformation, which would have been better called inversion.

It is a lot of work to get all the conservation laws by Noether's theorem; they were rarely first found that way, and not all of them are useful. It is easier to use generators or multipliers or what Friedrichs called the abc method. For example, we get conservation of energy by multiplying $\square u$ by $u_{t}$ and integrating by parts.

Thus

$$
u_{t} \square u=\frac{1}{2}\left(u_{t}^{2}+|\nabla u|^{2}\right)_{t}-\operatorname{div}\left(u_{t} \nabla u\right) .
$$


Integrating over a slab from 0 to an arbitrary time, one sees that the integral of the energy density, $\frac{1}{2}\left(u_{t}^{2}+|\nabla u|^{2}\right)$, does not change in time if the solution vanishes far out. This is the most used of the conservation laws.

Next comes the conservation law that we obtain under a conformal transformation. First we take the transformation,

$$
\begin{aligned}
& x, t, u \rightarrow X, T, U \\
& |x|=r \text { and }|X|=R \\
& (x, t)=(X, T) /\left(T^{2}-R^{2}\right) \text { i.e. }(X, T)=(x, t) /\left(t^{2}-r^{2}\right),
\end{aligned}
$$

and, with $m$ the number of space dimensions,

$$
r^{\frac{m-1}{2}} u=R^{\frac{m-1}{2}} U
$$

Then

$$
\begin{aligned}
\square u= & \text { Factor } \\
& \times \square U .
\end{aligned}
$$

The easy way to check this invariance is to set $r^{\frac{m-1}{2}} u=R^{\frac{m-1}{2}} U=W, t+r=$ $1 /(T-R)=\xi=1 / \Xi$, and $t-r=1 /(T+R)=\eta=1 / H$.

In the end, we obtain

$$
\left(\left(t^{2}+|x|^{2}\right) u_{t}+2 t x \cdot \nabla u+(m-1) u\right) \square u=Q+\operatorname{div} P,
$$

which is just the energy identity for $U$.

The strength of this identity is that the contribution from any space-like surface, for example, $t=$ constant, has a definite sign because the image of the surface in $(X, T)$ space is also space-like.

The first example [13] of its use is for a solution of the wave equation in the exterior of a star-shaped obstacle, on which the solution vanishes. Integrating by parts, again in the slab $\left(0<t<t_{1}\right)$, one obtains a bound for the integral on $t=t_{1}$ in terms of the initial data. The term from $Q$ can be rewritten as integrals with positive integrands. Thus,

$$
\int P \cdot n d S+\left.\int q d x d y d z\right|_{t=T}=\left.\int q d x d y d z\right|_{t=0}
$$

where $q=q_{1}+q_{2}+q_{3}$ and

$$
\begin{aligned}
& q_{1}=\left(r^{2}+t^{2}\right)\left(|\nabla u|^{2}-u_{r}^{2}\right) \\
& q_{2}=\frac{1}{4 r}(r+t)^{2}\left((r u)_{r}+r u_{t}\right)^{2} \\
& q_{3}=\frac{1}{4 r}(r-t)^{2}\left((r u)_{r}-r u_{t}\right)^{2}
\end{aligned}
$$

and the term in $P$ is

$$
\int t\left(\frac{\partial u}{\partial n}\right)^{2} \vec{x} \cdot \vec{n} d S d t
$$

over the obstacle. Here $\vec{n}$ is the normal derivative. Star-shaped means $\vec{x} \cdot \vec{n} \geq 0$.

Every term is nonnegative, so each is bounded, and thus the first term yields the decay of the $L^{2}$ space norm of the angular derivatives, in fact, like $l / t$. The 
second shows that the $L^{2}$ space norm of the derivative in the outward characteristic direction also decays like $l / t$. These ultimately yield Sommerfeld's radiation condition.

The remaining derivative in the inward direction decays like $l / t$ only in a local $L^{2}$ norm.

That is the best, there are in $2 \mathrm{D}$ for obstacles that do not trap rays. In $3 \mathrm{D}$, Huyghens principle will give you exponential decay, but that is a long story; see the book by Lax and Phillips [8].

Not surprisingly, there are analogous formulas for the reduced wave equation in the exterior of a star-shaped obstacle. These give estimates for the error created by high-frequency approximation [10] (geometrical optics). The formulas were found by hunting and fishing for a workable multiplier analogous to the multiplier of (3.2).

Decay of a similar kind can also be proved for semilinear equations of the form:

$$
\square u+F^{\prime}(u)=0
$$

with $F^{\prime}(u)=d F(u) / d u$ satisfying some conditions beyond making the energy contribution from $F^{\prime}(u)$ positive, that is, $F(u) \geq 0$.

Of course the conformal transformation does not lead to a conservation law. The extra terms in the previous divergence equation are

$$
\left(\left(t^{2}+r^{2}\right) F(u)\right)_{t}+t\left((m-1) u F^{\prime}(u)-2(m+1) F\right) .
$$

To get decay, the second term, which gets integrated over space and time, must be nonnegative, which forces $F$ to satisfy

$$
(m-1) u F^{\prime}(u)-2(m+1) F \geq 0 .
$$

As a result you cannot get decay if, say, $F(u)=u^{2}+u^{4}, m=3$, the semilinear example, the nonlinear Klein-Gordon equation, first proposed by Heisenberg. For that case a very weak decay can be obtained, with a different multiplier [14], that can be jacked up eventually to give a scattering theory for the equation [15]. A scattering theory tells us the relationship between the solution at $t=-\infty$ and the solution at $t=+\infty$.

Strichartz [21], followed by Ginibre and Velo [5], altered the art of finding decay for semilinear equations, obtaining uniform Sobolev space estimates in many cases. So multipliers and invariants do not have all the answers.

On the other hand, conservation identities that were used originally for decay provide an unusual and useful handle for some otherwise unobtainable local estimates, as Shatah and Struwe have done in their studies of regularity for semilinear problems in the so-called critical case, critical because standard Sobolev inequalities do not help. Thus

$$
F^{\prime}(u)=u|u|^{p-2}
$$

with $p=2 m /(m-2)$. See also Bourgain [1] for the corresponding nonlinear Schrödinger equation.

We get the flavor of their method in the critical case and consider $u$ real and positive for convenience.

Here is the problem. We can solve (3.3), (3.5) for short times as a $C^{\infty}$ solution if the Cauchy data on $t=0$ are $C^{\infty}$. But does the solution remain so regular for all times? If not, there is a first time $t_{0}$ when the regularity ceases. This could be at many different points in space. Let one of them be the origin. The "solution" at $\left(0, t_{0}\right)$ depends on the characteristic cone drawn backwards in time, and it is 


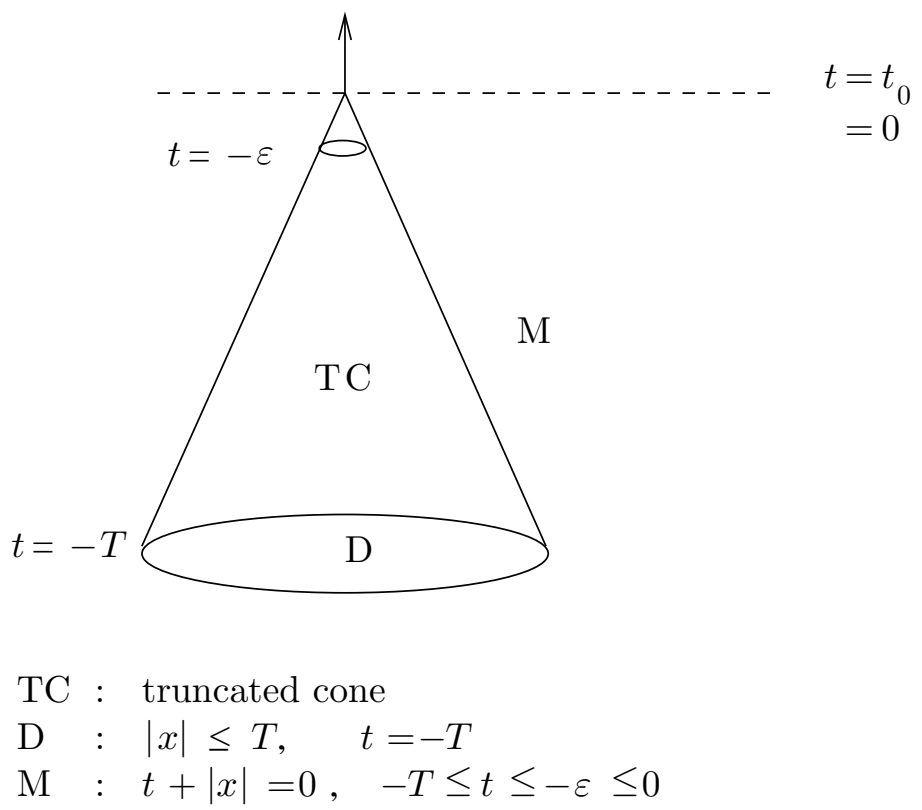

\section{FiguRE 3}

from properties in that cone combined with the multiplier method that we find a very minimal regularity inside the cone as we approach $\left(0, t_{0}\right)$. That minimal regularity is described as follows; see Figure 3. Let $C$ be the backward half cone $t_{0}-t>\left|x-x_{0}\right|$, and let $\mathcal{M}$ be its mantel $t_{0}-t=\left|x-x_{0}\right|$. We slice the cone into a truncated cone, $T C$, by $t_{0}-t=T$ and $t_{0}-t=\epsilon$ where $T>0$, and $\epsilon$ will be smaller than anything. The standard energy identity can be applied to $T C$ since the solution is regular for $t<t_{0}$.

Take $t_{0}=0$. Then (a subscript $i$ on $u$ means a derivative),

$$
\begin{aligned}
0 & =u_{t}\left(u_{t t}-u_{i i}+u^{p-1}\right) \\
& =\left(\frac{u_{t}^{2}}{2}\right)_{t}-\left(u_{t} u_{i}\right)_{i}+\left(\frac{u_{i} u_{i}}{2}\right)_{t}+\left(\frac{u^{p}}{p}\right)_{t},
\end{aligned}
$$

or integrating over the cone,

$$
\begin{aligned}
0= & \int_{\partial \Omega}\left(\frac{u_{t}^{2}}{2} t_{n}-u_{t} u_{i} x_{i n}+\frac{u_{i} u_{i}}{2} t_{n}+\left(\frac{u^{p}}{p}\right)\right) d S \\
= & \int_{\text {mantel of TC }}\left(\left(\frac{u_{t}-u_{r}}{2}\right)^{2}+\left(\frac{|\nabla u|^{2}-u_{r}^{2}}{2}\right)\right) d \widetilde{S}+\frac{u^{p}}{p} \frac{d \widetilde{S}}{\sqrt{2}} \\
& +\left[\int\left(\frac{1}{2}\left(u_{t}^{2}+|\nabla u|^{2}\right)+\frac{u^{p}}{p}\right)|d x|\right]_{t=T}^{t=\epsilon}
\end{aligned}
$$

where $d \widetilde{S}$ is an appropriate surface element on the cone $r+t=0$.

Thus not only is the energy bounded by its initial value, but the flux across the mantel of $T C$ which is monotonically increasing is also uniformly bounded 
and hence has a limit. Thus we can turn the argument around and say that for $T$ sufficiently small, the flux across the mantel of $T C$ is $o(1)$ in terms of the energy. We note for further use that if this expression is rewritten in terms of $w=r^{\frac{m-1}{2}} u$, one can integrate the indefinite terms in $w w_{r}$ where $r=|x|$ and find that $\int_{\text {mantel of } T C}\left(w_{t}-w_{r}\right)^{2} d r d \sigma$ is also $o(1)$ where $d r d \sigma$ is the surface element in polar coordinates on the cone. This can easily be recognized from the spherically symmetric case. Using $w=r^{\frac{m-1}{2}} u$ and Schwarz' inequality on the flux form in $w$ and the flux form in $u$, one finds $\int u^{2} r^{-2} d S$ is also $o(1)$. The energy on $t=\epsilon$ is, however, by this kind of argument only bounded. Some of the energy may vent through the tip of the cone, and we cannot eliminate this by energy consideration alone. Further

$$
\int_{t=-\epsilon}\left\{r^{-m+1}\left(w_{t}^{2}+w_{r}^{2}\right)+\left(|\nabla u|^{2}-u_{r}^{2}\right)+\frac{u^{p}}{p}\right\}|d x|
$$

is bounded by the energy at $t=T$.

The precise result we are after is that

$$
\lim _{T \rightarrow 0} \int_{|x|<-T} u^{p}|d x|=0 .
$$

This is a very weak result. But Shatah and Struwe [20] show that it can be jacked up to show that the solution can be continued as a regular solution beyond 0 , in particular in the critical case.

To prove (3.6), we use an estimate obtained by employing the multiplier ${ }^{1} t u_{t}+$ $x \cdot \nabla u+\frac{m-1}{2} u$.

The easiest way to obtain the most useful identity is to compute it the oldfashioned way with subscripts. However, before plunging in, we point out that a more elegant way is to notice that a shift in $t$ would take us from the multipliers of (3.2) to the multiplier $x \cdot \nabla u+t u_{t}+\frac{m-1}{2} u$ and that the identity we obtain must be the same as the identity obtained by shifting $t$ in the old identity. Then we would collect terms to get definite expressions. Here we work directly. Thus

$$
\begin{aligned}
0 & =\left(t u_{t}+x_{j} u_{j}+\frac{m-1}{2} u\right)\left(u_{t t}-u_{i i}+u^{p-1}\right) \\
& =\bar{Q}_{t}+\bar{P}_{i i}+\bar{R}
\end{aligned}
$$

with

$$
\begin{aligned}
\bar{Q} & =\frac{1}{2} t u_{t}^{2}+\frac{1}{2} t u_{i} u_{i}+x_{j} u_{j} u_{t}+\frac{m-1}{2} u u_{t}+\frac{t u^{p}}{p} \\
\bar{P}_{i} & =-t u_{t} u_{i}-\frac{1}{2} x_{i} u_{i}^{2}-x_{j} u_{j} u_{i}+\frac{1}{2} x_{j} u_{j} u_{j}-\frac{m-1}{2} u u_{i}+\frac{1}{\rho} u^{p} x_{i} \\
\bar{R} & =\left(-\frac{(1+m)}{p}+\frac{m-1}{2}\right) u^{p} .
\end{aligned}
$$

We note that in the critical case $\bar{R} \geq 0$.

\footnotetext{
${ }^{1}$ This multiplier was used in finding decay of energy near an obstacle [13] and not as it is frequently referenced in the paper [14] on the Klein-Gordon equation.
} 
The next step is a slightly tricky rearrangement to absorb the indefinite terms like $\frac{m-1}{2} u u_{i}$ using $\frac{x_{i} u_{i}}{r}=u_{r}$ and $w=r^{\frac{m-1}{2}} u$; then

$$
\begin{aligned}
\bar{Q}=\frac{1}{2} t\left(|\nabla u|^{2}-\right. & \left.u_{r}^{2}\right) \\
& +\frac{1}{4}(t+r)\left(u_{t}+u_{r}\right)^{2}+\frac{1}{4}(t-r)\left(u_{t}-u_{r}\right)^{2}+\frac{m-1}{2} u u_{t}+\frac{t u^{p}}{p} \\
=\frac{1}{2} t\left(|\nabla u|^{2}-\right. & \left.u_{r}^{2}\right) \\
& +\frac{1}{4}(t+r) r^{-m+1}\left(w_{t}+w_{r}\right)^{2}+\frac{1}{4}(t-r) r^{-m+1}\left(w_{t}-w_{r}\right)^{2}+\frac{t u^{p}}{p} .
\end{aligned}
$$

We integrate over the truncated cone $T C$. It is now quite easy to see from the second form of $\bar{Q}$ that we may let $\epsilon \rightarrow 0$ using the energy bounds of the previous argument.

The rest of the argument follows by showing that the integral over the mantel is $T o(1)$. This is because the integral over the base, $t=T$, has the same sign as the volume term (second form of $\bar{Q}$ ); hence the integral over the base is $T o(1)$. Further, each term in $\bar{Q}$ is nonnegative. Hence $\int T u^{p}|d x|$ is $T o(1)$, and thus $\int u^{p}|d x|$ is $o(1)$.

All that is left is to check that the integral over the mantel is $T o(1)$. The integral over the mantel where $d t=-d r$ is:

$$
\int\left(\bar{Q}+\bar{P}_{i} \frac{x_{i}}{r}\right) d r d \sigma
$$

where again $d r d \sigma$ is a surface element. We note that

$$
\begin{aligned}
\bar{P}_{i} \frac{x_{i}}{r} & =-t u_{t} u_{r}-\frac{1}{2} r u_{t}^{2}-r u_{r}^{2}+\frac{1}{2} r|\nabla u|^{2}-\frac{m-1}{2} u u_{r}+\frac{r}{p} u^{p} \\
& =-\frac{1}{2} t\left(|\nabla u|^{2}-u_{r}^{2}\right)+\frac{t}{2}\left(u_{t}^{2}-u_{r}^{2}\right)-t u_{t} u_{r}-\frac{m-1}{2} u u_{r}-\frac{t}{p} u^{p} .
\end{aligned}
$$

Thus $\int_{\text {mantel }}\left(\bar{Q}+\frac{\bar{P}_{i} x_{i}}{r}\right) d r d \sigma$, by using the bounds on the flux across the cone in terms of $u$, is $T o(1)$.

We have utilized here the bound on the integral $u^{2} / r^{2}$ on the cone that the second energy form gives. Note that the term in $\frac{u^{p}}{p}$ and the term involving $u_{t}+u_{r}$ drop out.

This completes the argument. Jacking the result up to full regularity is complicated, and we do not try to do that here.

\section{BLACK HOLES}

I turn now to my last topic, black holes. What does that have to do with conservation laws and the wave equation? They are related, but frankly this last topic came about because when all the world was telling me what to talk about on this occasion, someone said, "black holes." And I thought, "It doesn't fit my title, and I have forgotten what they really are." But I went home and looked in the encyclopedia which sits in our living room. (It is very beneficial for resolving domestic disputes to have an encyclopedia in the living room.) I looked up "black holes". There I learned of the three ways in which a star can die.

Most stars are composed of hydrogen. But let me digress a moment. That most stars are hydrogen was discovered by Cecilia Payne [18] through spectral analysis 
at Harvard in 1923, but the big-shot astronomers pooh-poohed it and talked her out of her convictions. She wrote her thesis up as a "maybe" and frankly did not get the credit until long after the truth had become evident.

But back to the death of stars. Most stars make energy by converting their hydrogen into helium and down the periodic table into other elements. The first kind of star is small, the process stops and the star becomes a "white dwarf". "Small" means having a mass like our sun's. The second kind of star, postulated by Chandrasekhar and much bigger, becomes a "supernova" and explodes its mass into the universe. The pressure beats the gravitational pull. The third kind of star (about fifty solar masses) turns into iron, and there is nowhere to go. Further fusion would not release more energy. Again the pressure of the gas competes with the gravitational pull to implode. The speeds and forces tell us that the phenomenon is to be treated by general relativity, and that is where the wave operator comes in.

The bending and twisting of the characteristics of the system give the gravitational field and, despite the countervailing pressure of the gas, create a black hole into which everything falls and from which no signals emerge. It appears to have been so named by John Wheeler, and the name alone excites everyone's fancy.

That is more or less what the encyclopedia said. Looking for more, I moved on to a popular book, Black Holes and Time Warps, by Kip Thorne [23]. I was reminded of Alice in Wonderland.

"What is the use of a book," thought Alice, "without pictures or conversation?" Thorne's book had plenty of pictures and conversation. In fact, it's very good reading. But this Alice is a mathematician and my thought was, "What is the use of a book in science without equations or formulas?" So I turned to more mathematical sources, mostly friends. I particularly want to acknowledge my late father, John L. Synge; Martin Kruskal; Satya Kichenessamy; Jalal Shatah; and Demetrious Christodoulou.

The problem of the black hole actually goes back to the end of the eighteenth century, when many believed that light was composed of corpuscles of mass subject to gravity. John Michell [11] (like many other rectors in the Church of England, he preached on Sunday and did science the rest of the week) determined the radius of a star from which light cannot leave because the velocity of light (known with some accuracy at the time) is less than the escape velocity. Remember, that is the velocity a vehicle needs to be able to escape the gravitational field of a star. Stars from which light could not escape were called "dark stars". That was two hundred years before relativity.

The first relativistic model for a dark star was proposed by Schwarzschild [19] in 1916 in two papers written on the Eastern front, where he was serving in the German army. (He died of illness soon after.) His model was imperfect, and there was a deep underlying problem.

But now this Alice is sinning! We need some equations and formulas. We begin with the metric in space-time:

The Einstein metric is given by

$$
d s^{2}=g_{\alpha \beta} d x^{\alpha} d x^{\beta} \quad\left\{g_{\alpha \beta}\right\}=G, \quad \alpha \beta=0,1,2,3 .
$$

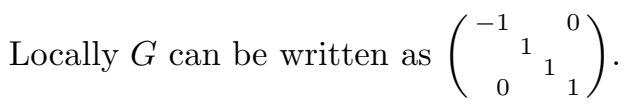


The Einstein equations are

$$
E(G)=T \text {. }
$$

The left hand side, $E(G)$, is geometric; it is associated with curvature in space-time. $T$ is a tensor which governs the gas dynamics. The operator $E$ may be written as

$$
E=\square G+Q_{1}(G)+Q_{2}(G) .
$$

At last we see the wave operator, followed by $Q_{1}$ and $Q_{2}$, which are nonlinear. They depend on $G$ and its first two derivatives. $Q_{1}$ is quasilinear - that is, it is linear in the second derivatives of $G$-and $Q_{2}$ is quadratic in the first derivatives.

In the Einstein equations, geometry is separated from the gas dynamics, but there are a lot of unknowns. This is already a mess, and there are also compatibility conditions. But as it turns out the count is right for the system of p.d.e.

We pass over the famous work on the initial value problem by Yvonne Choquet Bruhat [2] and the more recent work of Christodoulou and Klainerman [4] and many others.

What are we looking for when we look for a black hole? For a black hole we need a solution to these equations, that is, a metric plus gas variables (we do have to put in some gas laws) that form a solution in the weak sense starting from some initial manifold where we may say these equations take over from some sort of fiery boundary layer. But this solution must FAIL at some later time - that is the HOLE. All particle paths must fall into it. To be BLACK we have to be able to say we cannot see into it; that is, we cannot receive a signal from it.

Let us try for a simple case - spherical symmetry. The metric will then have the form:

$$
d s^{2}=g_{11} d r^{2}+2 g_{12} d r d t+g_{22} d t^{2}+r^{2}\left(d \theta^{2}+\sin ^{2} \theta d \phi^{2}\right) .
$$

Case 1. The star is a point mass. The metric is the Schwarzchild metric:

$$
d s^{2}=\left(1-\frac{2 m}{r}\right)^{-1} d r^{2}-\left(1-\frac{2 m}{r}\right) d t^{2}+r^{2}\left(d \theta^{2}+\sin ^{2} \theta d \phi^{2}\right) .
$$

This is certainly expected to describe the metric at large distances.

Here $m$ is the mass of the star. There are two singularities:

$$
\begin{aligned}
& r=2 m, \\
& r=0 .
\end{aligned}
$$

It is clearly all right to have a singularity at $r=0$, but what about $r=2 m$ ? It turns out that this is not a real singularity and can be fixed by making a change of the independent variables.

My father, John L. Synge [22], in a paper presented to the Royal Irish Academy in 1949, following Lemaitre [9] and H.P. Robertson, constructed a change of variables that continued the metric smoothly and where a full description could be made of the paths of particles in space-time not only near $r=2 \mathrm{~m}$ but also near the true singular point $r=0$. A better change of variables by Martin Kruskal [7] - that is, a better mapping - fixed the remaining defects of the Schwarzschild metric.

As a last stop on the black hole story, I would like to describe the results of the model of Christodoulou [3], who began this work more than 10 years ago. He uses a gas with a somewhat strange gas law. It is due to Oppenheimer [17] and has been 
used by Bethe and others. Don't forget this gas is a very unusual end product of a long process.

Again under spherical symmetry, the metric may be put in the form

$$
-\Omega^{2} d u d v+r^{2}\left(d \theta^{2}+\sin ^{2} \theta d \phi^{2}\right) .
$$

$u=$ constant represents a light ray moving out with fixed latitude and longitude; $v=$ constant correspondingly moving in.

We have to find $r$ and $\Omega$ as functions of $u$ and $v$. It is easier to work with the mass-energy density $m$ instead of $\Omega$. But they are simply related:

$$
\Omega^{2}\left(1-\frac{2 m}{r}\right)=-4 r_{u} r_{v} .
$$

The equations reduce to

$$
\begin{gathered}
r r_{u v}=(2 m / r)\left(1-\frac{2 m}{r}\right)^{-1} r_{u} r_{v} \\
r_{u} m_{u}=2 \pi\left(1-\frac{2 m}{r}\right) r^{2} \Phi_{u}^{2} \\
r_{v} m_{v}=2 \pi\left(1-\frac{2 m}{r}\right) r^{2} \Phi_{v}^{2} \\
r \Phi_{u v}+r_{u} \Phi_{u}+r_{v} \Phi_{u}=0
\end{gathered}
$$

Note the wave operator appearing here in characteristic form. And also note that the equations are clearly singular for $r=0$ and for $r=2 m$. But again the results show that only $r=0$ is a genuine singularity.

Besides $r$ and $m$ we now have $\Phi$. This comes from the gas model. It makes it appear that there are 4 equations for 3 unknowns, but computation shows that the last equation is the compatibility condition that $m$ satisfy the two preceding ordinary differential equations on the characteristics.

Christodoulou [3] has proved the existence of solutions of bounded variation that are unique. Data is specified for $m$ and $r$ on $u=0$, let us say, and because of spherical symmetry on $u-v=0$.

A rather mild condition must be imposed on the data in order to end up with a black hole.

Christodoulou makes the figure (see Figure 4) with the $u, v$ axes rotated through $45^{0}$.

Along with the $u, v$ space there is the spherical coordinate space $\theta, \phi$, and so we are only interested in the right half plane and have $r=m=0$ on the axis.

There is a singularity for all of these solutions where $r=0, m>0$. Various derivatives blow up. This is the HOLE.

There is a second curve, $A$, where $r=2 m$. The solution can be continued across it, but every world path then proceeds into the hole. It forms what Christodoulou calls an apparent horizon. Notably it is asymptotic as $v \rightarrow \infty$ to a characteristic (a light ray) $u=$ constant. That is $H$. It is really a cone in the 4 -space. It contains the paths of the last signals that can go off to $\infty$. This makes the hole BLACK. No light paths escape beyond it to $\infty$. 


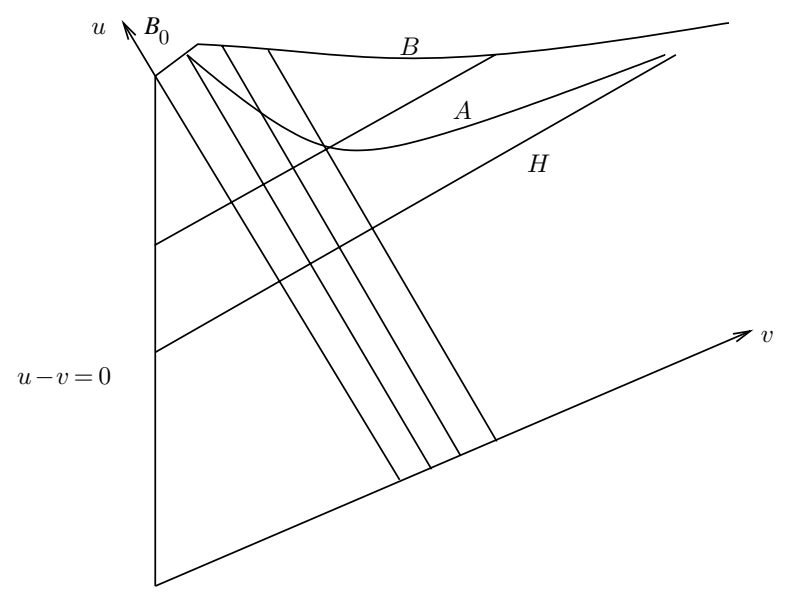

FiguRe 4

Much more could be said about the mathematics of black holes. Physicists have explored the interaction of, say, two black holes. Where does that fit in? And so on.

But where are the conservation laws, and where are the invariants?

The equations are invariant under a change of scale, but they do not seem to have any conservation laws (nor should they). In fact, the change of scale invariance makes it harder to make estimates instead of easier.

The space of Bounded Total Variation comes naturally from 1D-time gas dynamics, but it is not right there for more space variables, so we do not expect it to be right here if we abandon spherical symmetry. We know $L^{2}$ estimates are no good for these problems even for small data.

So here is the wave equation - in desperate need of new estimates, for the world is not likely to be spherically symmetric. And that is where we will leave it. Black holes bring us to the wave operator, but not to its conservation laws.

\section{REFERENCES}

1. Bourgain, J. Global well-posedness of defocusing critical nonlinear Schrödinger equation in the radial case. Journal of the A.M.S., 12, no. 1, 145-171, January 1999. MR 99e:35208

2. Choquet-Bruhat, Y. Theorème d'existence pour certain systemes d'equations aux derivées partielles nonlineaires. Acta Mathematica, 88 (1952), 141-225.

3. Christodoulou, D. The mathematical theory of gravitational collapse, in Current Trends in Applied Mathematics Editoriale COMPLUTNSE, Universitat, ISPN 84-9365-94-6. Editors: M. A. Herrero and E. Zuazua, 1996.

See also for essentially the same picture, Formation of black holes and singularities in spherically symmetric grativitational collapse, Comm. Pure Appl. Math. 44 no. 3, (1991), 339-373. MR 92d:83044

And for the bounded variation results see, Bounded variation solutions of the spherically symmetric Einstein-scalar field equations, Comm. Pure Appl. Math. 46 (1993), 1131-1220. MR 95b:35221

4. Christodoulou, D. and Klainerman, S. The global linear stability of the Minkowski Space, Princeton Mathematical Series 41, Princeton University Press, 1993. MR 95k:83006

5. Ginibre, J. and Velo, G. The global Cauchy problem for the nonlinear Klein-Gordon equation. Math. Zeit. 189 (1985), 487-505. MR 86f:35149

6. Heisenberg, W. Mesonerzeugung als Stosswellen problem. Zeit. Physik 33 (1952), 65-79. 
7. Kruskal, M.D. Maximal extension of Schwarzschild Metric. Phys. Rev. 119, no. 5 (1960). MR 22:6555

8. Lax, P.D. and Phillips, R.S. Scattering Theory, Pure and Appl. Math., Academic Press, New York, 1967. MR 36:530

9. Lemaître, G. L'univers en expansion, Ann. Soc. Sci. Bruxelles A 53 (1933), 51-85.

10. Ludwig, D. and Morawetz, C.S. An inequality for the reduced wave operator and the justification of geometrical optics. Comm. Pure and Appl. Math. 21 (1968), 187-203. MR 36:6185

11. Michell, J. On the Means of Discovering the Distance, Magnitude, Etc. of the Fixed Stars, in Consequence of the Diminution of their Light, in Case such a Dimunution Should be Found to Take Place in Any of Them and Such Other Data Should be Procured from Observations as Would be Further Necessary for That Purpose, Phil. Trans. of RS of London 74 (1784), 55, presented 1783 .

12. Morawetz, C.S. Note on a maximum principle and a uniqueness theorem for an elliptichyperbolic equation. Proc. Roy. Soc., A, 236 (1956), 141-144. MR 18:133a

13. Morawetz, C.S. The decay of solutions of the exterior initial-boundary value problem for the wave equation. Comm. Pure and Appl. Math. 14 (1961), 561-569. MR 24:A2744. See also, The Limiting Amplitude Principle, Comm. Pure and Appl. Math. 15 (1962), 349-361. MR 27:1696

14. Morawetz, C.S. Time decay for the nonlinear Klein-Gordon equation, Proc. Roy. Soc. A 306 (1968), 291-296. MR 38:2455

15. Morawetz, C.S. and Strauss, W. Decay and scattering of solutions of a nonlinear relativistic wave equation. Comm. Pure and Appl. Math. 25 (1972), 1-31. MR 46:2239

16. Noether, E. Invarianter beliebiger Differentialausdrücke. Nachr.Ges.d.Wiss.zn Göttingen (Math.Phys. Kl.), 37-44, (1918). Collected papers pp. 240-257.

17. Oppenheimer, J.R. and Snyder, H. On continued gravitational contraction. Phys. Rev. 56 (1939), 455.

18. Payne, C.H. Stellar Atmospheres: A Contribution to the Observational Study of High Temperatures in the Reversing Layers of Stars. Harvard Observatory, 1925.

19. Schwarzschild, K. Sitzber. Preuss. Akad. Wiss. Physik-Math. Kl. 189 (1916).

20. Shatah, J. and Struwe, M. Regularity results for nonlinear wave equations. Annals of Mathematics, 138 (1993), 503-518. MR 95f:35164

21. Strichartz, R.S. Restrictions of Fourier transforms to quadratic surfaces and decay of solutions of wave equations. Duke Math. Journal, 44 (1977), 705-714. MR 58:23577

22. Synge, J.L. The gravitational field of a particle. Proc. Roy. Irish Acad. A 53(1950), 83. MR 12:546g

23. Thorne, Kip S. Black holes and time warps: Einstein's outrageous legacy. New York, W.W. Norton (1994). MR 95h:83065

Courant Institute of Mathematical Sciences, 251 Mercer Street, New York, New YORK 10012

E-mail address: morawetz@cims.nyu.edu 